\title{
Applicability of a Classical Perturbation Technique for Perturbation Parameters with Large Values
}

\author{
Igor V. Andrianov \\ Institut für Allgemeine Mechanik, RWTH Aachen University,Templergraben 64, D-52056, Aachen, Germany \\ Jan Awrejcewicz
}

Technical University of Łódź, Department of Automation and Biomechanics, 1/15 Stefanowski St., PL-90-924, Łódź, Poland

(Received 30 December 2008; revised 27 May 2009; accepted 10 June 2010)

In this letter we will illustrate and discuss some problems regarding the validity and accuracy of the perturbationlike methods applied to systems with weak and strong non-linearities.

$\mathrm{Hu}^{1}$ studied the well-known Duffing equation:

$$
x^{\prime \prime}+\omega_{0}^{2} x+\varepsilon x^{3}=0
$$

which has the initial conditions of

$$
x(0)=A, x^{\prime}(0)=0 .
$$

$\mathrm{Hu}^{1}$ assumed the solution of Eq. (1) in the form of

$$
x(t)=x_{0}(t)+\varepsilon x_{1}(t)+\varepsilon^{2} x_{2}(t)+\cdots .
$$

The fundamental frequency $\omega^{2}$ is given by

$$
\omega^{2}=\omega_{0}^{2}+\varepsilon \omega_{1}+\varepsilon^{2} \omega_{2}+\cdots
$$

Notice that in classical approaches of the theory of perturbation $^{7}$ an asymptotic series of $x(t)$ is sought in Eq. (3), but the fundamental frequency being sought is estimated through the following equation

$$
\omega=\omega_{0}+\varepsilon \omega^{(1)}+\varepsilon^{2} \omega^{(2)}+\cdots
$$

instead of being estimated by using Eq. (4).

Equations (1) and (2) possess an exact solution, and hence a comparison of accuracy of Eqs. (4) and (5) can be carried out. Hu has shown numerically that Eq. (3), contrary to traditional application of Eq. (5), yields suitable results even for $0 \leq \varepsilon \leq \infty$.

$\mathrm{Hu}$ claims that he has derived a new perturbation technique that is valid for large parameters. ${ }^{1}$ However, this should be treated rather as a particular case, and such a general statement for any other dynamical systems remains invalid. In order to explain the result obtained by $\mathrm{Hu}^{1}$ we will recall the exact formula in what follows:

$$
\omega^{2}=\frac{\pi^{2}}{4}\left(\sqrt{1+\varepsilon A^{2}}\right) / K(m)
$$

where

$$
\begin{gathered}
K(m)=\int_{0}^{\pi / 2}\left(1-m \sin ^{2} \theta\right)^{-1 / 2} d \theta \\
m=\frac{\varepsilon A^{2}}{2\left(1+\varepsilon A^{2}\right)} .
\end{gathered}
$$

Since the following approximation holds ${ }^{8}$

$$
\begin{aligned}
K(m)=\frac{\pi}{2}\left[1+\left(\frac{1}{2}\right)^{2} m\right. & +\left(\frac{1 \cdot 3}{2 \cdot 4}\right)^{2} m^{2}+ \\
& \left.+\left(\frac{1 \cdot 3 \cdot 5}{2 \cdot 4 \cdot 6}\right)^{2} m^{3}+\cdots\right]
\end{aligned}
$$

and since for $0 \leq m \leq \frac{1}{2}$, Eq. (9) is convergent with a speed of geometrical progression convergence.

On the other hand, a solution representation in Eq. (4) allows avoiding the occurrence of the development of the expression $\sqrt{1+\varepsilon A^{2}}$ into a series whose radius of convergence is bounded.

Owing to some of the results presented by $\mathrm{Hu}^{1}$ it is difficult to judge whether Eq. (4) has greater advantages when it is compared to Eq. (5) for cases different from these defined by Eqs. (1) and (2).

For instance, it is often required to study a non-autonomous Duffing equation of the form of

$$
x^{\prime \prime}+\omega_{0}^{2} x+\varepsilon x^{3}=F \cos \phi t,
$$

or the autonomous in the form of

$$
x^{\prime \prime}+\omega_{0}^{2} x+\varepsilon x^{\alpha}=0, \quad \alpha>0, \quad \alpha \neq 3,
$$

with attached boundary conditions (see (2)), or, finally, the equation

$$
x^{\prime \prime}+\omega_{0}^{2} x+\varepsilon x^{\alpha}+\varepsilon a x^{\beta}=0, \quad \alpha \neq \beta, \quad a \equiv \text { const },
$$

and many other similar problems.

The main aim of this letter is to warn researchers that the title of $\mathrm{Hu}^{1}$ promises more than has been shown.

In addition, let us give our point-of-view regarding the discussion included in Sanchez and He. ${ }^{3,4}$ Sanchez's remark that the amplitude of oscillation of the Duffing equation is badly approximated by the perturbation technique for parameters with large values is not true. In order to show our statement, one may consider Fig. 1 given by Sanchez, ${ }^{3}$ where initial condition $A=1$ is not satisfied. In Sanchez ${ }^{3}$ initial conditions (see 